\title{
The Role of Gross Motor Performance Towards Hand- Eye Coordination Among Girls Aged 7 Years Old in Malaysia Using Machine Learning
}

\author{
I M Puspitasari ${ }^{1 *}$, M R Abdullah ${ }^{2}$, N A Kamarudin ${ }^{3}$, Faiza Renaldi ${ }^{4}$ and \\ Esmeralda C. Djamal ${ }^{5}$
}

\author{
${ }^{1,3}$ Faculty of Applied Science Social University of Sultan Zainal Abidin, 21300, University of Sultan Zainal Abidin, Gong \\ Badak Campus, Terengganu, Malaysia \\ ${ }^{2}$ East Coast Environment Research Institute, 21300, University of Sultan Zainal Abidin, Gong Badak Campus, \\ Terengganu, Malaysia \\ ${ }^{4,5}$ Informatics Department, Universitas Jenderal Achmad Yani, Bandung Barat, Indonesia \\ *Corresponding author.Email: razali896@yahoo.com
}

\begin{abstract}
Range of motor performance is a prerequisite for children to participate in daily activities and has significant consequences for different aspects of children and adolescent's growth. The objective of the study is to clusterize the coordination performance, to validate the role of gross motor performance towards classification of hand-eyes coordination and to develop best fit model among girls aged 7 years old in Malaysia. The sample size of this study was calculated using $\mathrm{G}$ power software. The statistical power was set at $95 \%$ with an effect size of $0.50 \%$. According to [1], an effect size of +0.20 was considered as small, +0.50 as medium and +0.80 as strong. Population for this analysis were student of 7-year-old girls in primary schools in peninsular Malaysia and Borneo. In this study anthropometric measurement and motor performance data was collected from 23133 girls from all districts in Malaysia. Analysis that used in this research was univariate clustering, discriminant analysis and machine learning. Univariate clustering helps in grouping the coordination. Next, discriminant analysis helps in validating the group and shows the prediction that correctly assigned to the group. Lastly, machine learning help in developing best fit model. In conclusion, this research can help many parties in an effort to improve coordination among girl aged 7 years old in Malaysia.
\end{abstract}

Keywords: Gross motor performance, Hand-eye coordination, 7 years old girl, Machine Learning

\section{INTRODUCTION}

Mastery of a range of motor performance is a prerequisite for children to participate in daily activities and has significant consequences for different aspects of children and adolescent's growth [2]. Therefore, the study of the motor performance of a child is a prerequisite for a full understanding of the entire development of the child. It has also been suggested that increased physical activity can cause excitement and decrease boredom, resulting in increased span of attention and improved focus [3]. In fact, physical activity can increase self-efficacy and self-esteem feelings and can boost class behaviour [4].
Hand-eye coordination is the strength that helps the eyes to
direct the hands in exact movement [5]. Great hand-eye
coordination in so many different aspects of life will
support your child [6]. Hand-eye coordination will help
your child catch a pitch, strike a pitch with a bat, and
graduate to more demanding demands of sport. The
integration of visual motors, which is a critical basis for
handwriting, also grows out of eye-hand coordination [7].
The eyes need to direct the hand in forming the letters and
ensuring that they stay within the lines. Eye tracking skills
that are vital for reading can be established through the
games and exercises used to guide the hand-eyes. Young 
kids are using this experience to learn how to stack towers, build with Lego etc. Even when we tie shoelace, we need our eyes to direct our hands.

Hand-eye coordination, or eye-hand coordination, is the capacity to do tasks that allow our hands and eyes to be used simultaneously, such as an action that uses the information our eyes interpret (visual spatial perception) to direct our hands in moving [5]. Eye-hand coordination is a complex cognitive capacity, because it allows us to integrate our vision and motor performance so that the hand is directed by the visual stimuli our eyes receive [8]. Hand-eye coordination is especially important for normal child growth and academic performance, but is also a valuable ability that adults engage on a daily basis in countless activities [9]. Most of the activities you do in your daily life use some degree of eye-hand coordination, which is why making sure it is as developed as possible is important [10].

Children with motor performance disorders are considered to have cognitive difficulties and poor social competencies [11]. Studies have shown that poor motor coordination can influence children's sense of competence, peer success, academic achievement and even their choice of leisure activities [12];[13]. The link between performance skills and social or emotional functioning is generally regarded as indirect [5]. Bad motor performance in person and team games / sports can lead to poor results that can undermine the sense of competence of a child [14]. It in turn reduces achievement within peer groups, decreases academic performance [15], and increases the child's perception of anxiety and depression [16]-[18]. In addition, factors responsible for poor motor performance can influence social competence by reducing the child's ability to interpret signals that are socially important.

Children with concentration, motor performance, and perception disorders, according to Gillberg [19], typically have deficits in empathic ability that may account for impaired social functioning. Many of your everyday activities require some degree of eye-hand coordination, which is why it's crucial to make sure it's as good as possible [20]. In general, we use visual knowledge to correct an action that is not suitable for a situation, which is one of the reasons why this cognitive capacity is so necessary [21].

\section{MATERIAL AND METHOD}

\subsection{Area of study}

This study focused on gathering data from students of 7 year-old girls at primary schools in peninsular Malaysia and Borneo. Such schools include national schools (SK), religious schools (SA), national schools of the Chinese type $(\operatorname{SJK}(\mathrm{C}))$, and national schools of the Tamil type $(\mathrm{SKJ}(\mathrm{T}))$. There is a total of 23133 samples. In this study anthropometric measurement and motor performance data was collected from 23133 girls from all districts in Malaysia.

\subsection{Determination sample size}

The sample size of this study was calculated using $G$ power software as shown in Figure 1. The statistical power was set at $95 \%$ with an effect size of $0.50 \%$. An effect size of +0.20 was considered as small, +0.50 as medium and +0.80 as strong [1]. To test the result, level of significance 0.05 was selected and considered as sufficient for the study. In this study, an t-test was selected from the test family menu. Then, means between two dependent mean (matched pairs) was chosen from the statistical test menu. Then, a priori: compute required sample size $=$ given $\alpha$, power and effect size were selected for the type of power analysis. A total number of 54 girls were required in this study.

\subsection{Physical measurement}

2.3.1 Body Weight. The calculation of body weight results in the participants taking off their bulky outer clothing and shoes. The participant is in the middle of the table, so that the weight is equally distributed on both sides. The weights are moved before alignment of the arrows and weight is registered. The weight was measured using weight digital scale.

2.3.2 Height. Standing height is the average distance measured from the floor to the highest point on the back. The height of each participant was measured by using stadiometer. The participants' location faces straight ahead with shoes should be off, feet together, and arms on the sides. When the calculation is finished, knees, buttocks and upper back should also be in contact with the ground.

2.3.3 Speed. A timed $20 \mathrm{~m}$ sprint was used to calculate distance. The tests were performed on an indoor synthetic pitch, and the completion times were recorded using electronic timing gates. For a 90 second rest period they were repeated three times, and the best timing was taken to reflect the sprint results.

2.3.4 Power. Power component was tested with a standing broad jump. The participant tries to jump as far as possible while standing broad jump, landing on both feet without falling backwards. Three attempts are required, and consideration was given to the farthest.

2.3.5 Flexibility. The sit and reach were calculated to test the flexibility components. This test involves sitting straight down on the floor with legs extended. Feet with shoes off are set flat against the box with the soles, apart from the shoulder-width. The investigator holds both knees flat against the floor, if required. The participant reaches forward along the measuring line as far as possible with hands on top of each other and palms facing downwards. Upon completing three practice sessions, the fourth reach is kept for at least two seconds while measuring the distance. 
2.3.6 Hand-eye coordination. The hand wall toast test in the Bruininks-Oseretsky Test of Motor Proficiency Instrument, Second Edition (BOTMP-2) was carried out by following procedure. A mark was laid at a distance of one meter from the wall. Adhesive tape was used to form a rectangular square $(30 \mathrm{~cm}$ or $30 \mathrm{~cm})$ and as a target compartment was attached to the wall. The distance to the floor from the target compartment is one meter. The students stood behind the marked line during the test, and faced the wall. Students threw the tennis ball with one dominant hand into the target compartment, and caught it in both hands. Till ten attempts the tennis ball was pushed back against the wall. It registered the numbers of successfully captured throws.

\subsection{Data management}

This analysis contains 16,1931 sets of used data. Data from 23133 participants was composed of 7-year-old girl with 7 variables. Among the variables used are height, weight, BMI, standing wide jump, 20-m sprint, hand wall toasting and sit and reach. Once data analysis was performed, the data distribution was first evaluated. Data distribution is essential for deciding which data analysis should be used. For this analysis, the normality of data was calculated using Shapiro-Wilk and P-plot.

2.4.1 Pre-processing Data. It defined the preliminary procedure for analyzing data. Pre-processing of data related to sport requires the preparation and retrieval of data for review in the data mining room. Pre-processing may make them relevant, depending on the current and necessary format of the data and the research issue. The first step is pre-processing of data, which includes data cleaning and data reduction. Information cleaning is about treating incoherent information, noisy data and incomplete data.

The total missing data in the matrices were very small $(\sim 3$ $\%)$ compared to the overall data recorded. For the data analysis screening, the nearest neighbour method was applied using XLSTAT 2014 add-in software [22]. This method examines the distance between each point and the closest point to it. The nearest neighbour method is the simplest methods, where the end points of the gaps are used as estimates of all missing values [22]. The equation applied in this method is shown in Equation 1 below. Where $\mathrm{y}$ is the interpolant, $\mathrm{x}$ is the interpolant of the time point. Meanwhile, $\mathrm{y} 1$ and $\mathrm{x} 1$ are the range of coordinates for starting point of the gap, and the opposite for ending points of the gap is $\mathrm{y} 2$ and $\mathrm{x} 2$.

$$
\begin{aligned}
& \mathrm{y}=\mathrm{y} \_1 \text { if } \mathrm{x} \leq \mathrm{x} \_1+\left(\mathrm{x} \_2-\mathrm{x} \_1\right) / 2 \\
& \text { or } \\
& \mathrm{y}=\mathrm{y} \_2 \text { if } \mathrm{x} \leq \mathrm{x} \_1+\left(\mathrm{x} \_2-\mathrm{x} \_1\right) / 2
\end{aligned}
$$

2.4.2 Data Cleaning. The total collection of raw data is initially 49878 participating girls. This omitted the raw data with missing data, incomplete data and error data. Then only 35134 girls become the sum of the sample with complete results. The mean and standard deviation used to evaluate the cut off for the first past of the simple recursive outlier method was determined using the entire sample, among the full results. Comparison was made of the highest and lowest measurements against the cut-off values. Such extreme lower outer and extreme upper outer values have been excluded from the study since they fall below the measured limit [23]. Lastly, there are a total of 23133 samples left and classified as treated results.

\section{STATISTICAL ANALYSIS}

\subsection{Univariate clustering}

Cluster analysis (CA) was employed in this research to classify the coordinating pattern grouping. Univariate clustering allows to cluster observations according to a single quantitative variable [24]. Univariate clustering clusters $\mathrm{N}$ one-dimensional observations (described by a single quantitative variable) into $\mathrm{k}$ homogeneous classes [25]. The algorithm used here is very fast and uses the method put forward by W.D. Fisher [26]. This method can be seen as a process of turning a quantitative variable into a discrete ordinal variable. To maximize the homogeneity of the classes, we therefore try to minimize the sum of the within-class variances. CA is a suitable tool for defining and classifying components or subjects (observations / population) into clusters of greater class homogeneity and higher class heterogeneity with respect to a specified selection criterion [27]. In addition, Ward 's technique of using Euclidean distances as a degree of similarity in CA has proven a very thorough technique.

\subsection{Discriminant analysis (DA)}

Discriminant analysis is a common technique of explanatory and predictive data analysis, using a qualitative variable as output [28]. Discriminant Analysis (DA) is a statistical tool that can be used in explanatory or predictive systems such as testing a two- or threedimensional map whether the groups to which observations belong are distinct, displaying the group properties using explanatory variables, and predicting the group would belong to a new observation. Discriminant Analysis may be used in numerous applications, for example in ecology and the prediction of financial risks (credit scoring). For this analysis, DA was used to evaluate their respective classes on the basis of the mean variables and to use variables to predict group size. In this analysis, the DA was positioned in the raw data using normal, step-by - step and retrograde modes to discriminate against three classes of relative output (poor, moderate, and excellent) assigned by univariate clustering [29]. They were used to create DFs to determine differences in relative performance in the physical characteristics and variables of motor performance. The subjects' relative output was treated as dependent 
variables while all the measured components were treated as independent variables. Variables are counted step by step in the forward step mode, starting with the greatest significant variable until no significant adjustments have been obtained. In the step-by - step backward mode, variables are removed step by step starting with the less important variable until no substantial adjustments have been made.

\subsection{Machine learning}

Orange is an open source platform that provides inexperienced and experienced users with the machine learning and data visualization capabilities [30]. Orange is an open source visual programming software package based on modules, used for data visualization, machine learning, data mining, and data processing. Orange components are called widgets and range from basic visualization of data, selection of subsets, and preprocessing, to realistic evaluation of learning algorithms and predictive modelling. Machine learning is a data analytics tool which automates the building of analytical models [31]. Machine Learning method is way easier to use, low costs and can analyze more specific data samples. From the result of Discriminant Analysis, Machine Learning will establish group prediction and differentiate the F-value from the group. Machine learning also will notify the researcher which girl that have potential to move from actual group to predicted group.

\section{RESULT AND DISCUSSION}

\subsection{Univariate Clustering}

Univariate analysis is the simplest form of analyzing data. "Uni" means "one", so in other words your data has only one variable. It doesn't deal with causes or relationships (unlike regression) and its major purpose is to describe. It takes data, summarizes that data and finds patterns in the data. Univariate clustering allows to cluster observations according to a single quantitative variable. Some ways you can describe patterns found in univariate data include central tendency (mean, mode and median) and dispersion: range, variance, maximum, minimum, quartiles (including the interquartile range), and standard deviation.

The frequency for poor is 9112 with percentage $39.39 \%$ while the frequency of moderate is 7937 with percentage $34.31 \%$. Excellent category has the lowest frequency with the lowest percentage which is 6084 and $26.3 \%$. from this analysis, we can conclude that 7-year-old girls enrolled in the Malaysian and Borneo peninsular primary schools mostly in poor category.

Table 1 score for univariate clustering

\begin{tabular}{ccccccc}
\hline Status & Freq & Cum.Freq & $\%$ & Cum.\% & Group Range & Group \\
\hline 1 & 9112 & 9112 & 39.39 & 39.39 & $1 \leq$ Poor $\leq 3$ & Poor \\
4 & 7937 & 17049 & 34.31 & 73.7 & $4 \leq$ Moderate $\leq 6$ & Moderate \\
7 & 6084 & 23133 & 26.3 & 100 & $7 \leq$ Excellent $\leq 10$ & Excellent \\
\hline
\end{tabular}

\subsection{Discriminant Analysis (DA)}

Discriminant analysis is a popular explanatory and predictive data analysis technique that uses a qualitative variable as an output. Table 2 of this summary of classification table shows that 1575 observations from were correctly assigned to excellent group. However, 1084 observation has potential from excellent group were instead put into moderate group, and 23425 observations from excellent group were put into poor group. Therefore, 4509 of the observations from excellent group were incorrectly classified into other groups. Next, from the moderate group, there are 1271 observation were correctly assigned to moderate group. But then, there are 1232 observation that has potential to go to excellent group and 5434 observation go to 5434 to poor group. For this group, there are 6666 observation that incorrectly classified to another group. Lastly, there are 6974 observation that correctly assigned to poor group while 1022 observation incorrectly assigned to excellent group and 1116 observation to moderate group. However, since the correct percentage was low, we need to do discriminant analysis once again (Table 3 ) to make sure the justification correction at least $80 \%$ and above. The higher the justification correct, the more accurate chance of prediction. 
Table 2 confusion matrix for the cross-validation results

\begin{tabular}{cccccc}
\hline from $\backslash$ to & Excellent & Moderate & Poor & Total & \% correct \\
\hline Excellent & 1575 & 1084 & 3425 & 6084 & $25.89 \%$ \\
Moderate & 1232 & 1271 & 5434 & 7937 & $16.01 \%$ \\
Poor & 1022 & 1116 & 6974 & 9112 & $76.54 \%$ \\
Total & 3829 & 3471 & 15833 & 23133 & $42.45 \%$ \\
\hline
\end{tabular}

Table 3 confusion matrix for the second cross-validation results

\begin{tabular}{cccccc}
\hline from $\backslash$ to & Excellent & Moderate & Poor & Total & \% correct \\
\hline Excellent & 3292 & 495 & 40 & 3827 & $86.02 \%$ \\
Moderate & 15 & 2817 & 645 & 3477 & $81.02 \%$ \\
Poor & 0 & 32 & 15797 & 15829 & $99.80 \%$ \\
Total & 3307 & 3344 & 16482 & 23133 & $94.70 \%$ \\
\hline
\end{tabular}

\subsection{Machine Learning}

Machine learning was used in this study to classify and distinguish influences between groups or clusters. Orange software was implemented by selecting several machine learning processes such as Knn, Tree, SVM, Random Forest, Naïve Bayes Neural Network and Logistic Regression. The output of seven machine learning processes has shown in Table 4 that the Artificial Neural Network (ANN), Random Forest, Tree, Support Vector Machine (SVM), among others, analyses strong contrast. The Artificial Neural Network has the highest accuracy value (0.998) however. The performance of ANN models is evaluated through the area under curve (AUC), classification accuracy (CA), F1 rating, accuracy and recall. As shown in Figure 4, it could be observed that an area under the curve of 1,000 percent can be formed by the ANN. In addition, the classification accuracy efficiency model was 0.998 per cent for ANN. The results of the F1 performance in machine learning show that via ANN 0.998 per cent. F1 score is the equilibrated cost of accuracy and recall. The ANN accuracy results are 0.998 percent. Finally, the findings of the machine learning recall approach were 0.998 per cent for ANN. Table 5 shows the confusion matrix category of Machine Learning process approaches to cross-validation results. The output was applied on clusters identified by relative performance variation. The precision to use excellent, moderate and poor range. 99.9 percent excellent, 99.5 percent moderate and 99.9 percent low range respectively were for outstanding results. Excellent performance should be used as the indicator of moderate and poor range category classification to achieve girls aged 7 in Malaysia index. However, these components distinguish the groups from the related components analyzed in this analysis, based on their level of efficiency.

Table 4 Precision value in machine learning

\begin{tabular}{lccccc}
\hline Model & AUC & CA & F1 & Precision & Recall \\
\hline kNN & 0.994 & 0.950 & 0.950 & 0.951 & 0.950 \\
Tree & 0.982 & 0.974 & 0.974 & 0.974 & 0.974 \\
Random Forest & 1.000 & 0.996 & 0.996 & 0.996 & 0.996 \\
SVM & 0.987 & 0.928 & 0.928 & 0.928 & 0.928 \\
Naive Bayes & 0.946 & 0.859 & 0.852 & 0.848 & 0.859 \\
Neural Network & 1.000 & 0.998 & 0.998 & 0.998 & 0.998 \\
\hline
\end{tabular}


Table 5 Confusion matrix group of cross validation in machine learning Predicted

\begin{tabular}{lccccc}
\hline & & Excellent & Moderate & Poor & $\Sigma$ \\
\hline \multirow{3}{*}{ Actual } & Excellent & $99.9 \%$ & $0.1 \%$ & $0.0 \%$ & 3307 \\
& Moderate & $0.4 \%$ & $99.5 \%$ & $0.1 \%$ & 3344 \\
\hline & Poor & $0.0 \%$ & $0.1 \%$ & $99.9 \%$ & 16482 \\
\hline
\end{tabular}

\section{CONCLUSION}

This study will develop a new strategy to recognize coordination among 7-year-old girls as a result of the test. As an indicator, the best match model may be guidance for parents, teachers, and stakeholders to identify children according to their class and help improve their coordination, and indirectly help improve cognitive performance for their children. This is supported from a theoretical perspective in Piek et al., [32] findings indicate a close association between early gross motor performance and later school aged cognitive development, in particular speed processing and working memory. This finding confirms other researchers [33]-[35] who have argued that early experiences with locomotives are an important agent for change in progress, which should not be ignored when evaluating effective interventions.

\section{REFERENCES}

[1] J. Cohen, A power primer. Psychological bulletin, 112 (1) (1992) 155. DOI: https://doi.org/10. 1037/0033-2909.112.1.155

[2] L. Lopes, R. Santos, B. Pereira, V. P. Lopes, Associations between gross motor coordination and academic achievement in elementary school children. Human movement science, 32 (1) (2013) 920. DOI: https://doi.org/10.1016/j.humov.2012.05.005

[3] D. P. Coe, J. M. Pivarnik, C. J. Womack, M. J. Reeves, R. M. Malina, Effect of physical education and activity levels on academic achievement in children. Medicine and science in sports and exercise, 38 (8) (2006) 1515. DOI: https://doi.org/10. 1249/01.mss.0000227537.13175.1b

[4] A. B. H. M. Maliki, M. R. Abdullah, A. Nadzmi, M. A. R. Zainoddin, I. M. Puspitasari, N. F. A. Jibril, N. A. Nawi, S. M. Mat-Rasid, R. M. Musa, Z. Suhaili, N. A. Kamarudin, S. K. S. Ali, Kids motor performances datasets. Data in brief, 34 (2021) 106582. DOI: https:// doi.org/10.1016/j.dib.2020.106582
[5] D. H. Ballard, M. M. Hayhoe, F. Li, S. D. Whitehead, Hand-eye coordination during sequential tasks. Philosophical Transactions of the Royal Society of London. Series B: Biological Sciences, 337 (1281) (1992) 331-339. DOI: https://doi.org/10.1098/rstb.1992. 0111

[6] C. Yu, L. B. Smith, Hand-eye coordination predicts joint attention. Child development, 88 (6) (2017) 20602078. DOI: https://doi.org/10.1111/cdev.12730

[7] B. M. Bekker, A Comparison between five and six year old grade 1 children regarding their readiness for acquiring handwriting skills (Doctoral dissertation, University of Pretoria). 2013.

[8] G. Metta, G. Sandini, J. Konczak, A developmental approach to visually-guided reaching in artificial systems. Neural networks, 12 (10) (1999) 1413-1427. DOI: https://doi.org/10.1016/S0893-6080(99)00070-2

[9] L. Lauer, D. Gould, N. Roman, M. Pierce, How parents influence junior tennis players' development: Qualitative narratives. Journal of Clinical Sport Psychology, 4 (1) (2010) 69-92. DOI: https://doi.org/ 10.1123/jcsp.4.1.69

[10] B. L. White, P. Castle, R. Held, Observations on the development of visually-directed reaching. Child development, (1964) 349-364. DOI: https://doi.org/10. $2307 / 1126701$

[11] A. Cummins, J. P. Piek, M. J. Dyck, Motor coordination, empathy, and social behaviour in schoolaged children. Developmental Medicine \& Child Neurology, 47 (7) (2005) 437-442. DOI: https://doi.org/ 10.1111/j.1469-8749.2005.tb01168.x

[12] M. H. Cantell, M. M. Smyth, T. P. Ahonen, Clumsiness in adolescence: Educational, motor, and social outcomes of motor delay detected at 5 years. Adapted physical activity quarterly, 11 (2) (1994) 115-129. DOI: https://doi.org/10.1123/apaq.11.2.115 
[13] A. Losse, S. E. Henderson, D. Elliman, D. Hall, E. Knight, M. Jongmans, Clumsiness in children-do they grow out of it? A 10-year follow-up study. Developmental Medicine \& Child Neurology, 33 (1) (1991) 55-68. DOI: https://doi.org/10.1111/j.14698749.1991.tb14785.x

[14] S. Houwen, C. Visscher, K. A. Lemmink, E. Hartman, Motor skill performance of children and adolescents with visual impairments: A review. Exceptional Children, 75 (4) (2009) 464-492. DOI: https://doi.org/10.1177/001440290907500405

[15] R. F. Catalano, K. P. Haggerty, S. Oesterle, C. B. Fleming, J. D. Hawkins, The importance of bonding to school for healthy development: Findings from the Social Development Research Group. Journal of school health, 74 (7) (2004) 252-261.

[16] M. Francis, J. Piek, The effects of perceived social support and self-worth on depressive symptomatology in children with and without developmental coordination disorder (DCD). In Proceedings of the 38th APS annual conference. The Australian Psychological Society Ltd. (2003) 70-74.

[17] M. M. Schoemaker, A. F. Kalverboer, Social and affective problems of children who are clumsy: How early do they begin?. Adapted physical activity quarterly, 11 (2) (1994) 130-140. DOI: https://doi.org /10.1123/apaq.11.2.130

[18] R. A. Skinner, J. P. Piek, Psychosocial implications of poor motor coordination in children and adolescents. Human movement science, 20 (1-2) (2001) 73-94. DOI: https://doi.org/10.1016/S0167-9457(01)00 029-X

[19] C. L. Gillberg, The Emanuel Miller Memorial Lecture 1991: autism and autistic-like conditions: subclasses among disorders of empathy. Journal of Child Psychology and Psychiatry, 33 (5) (1992) 813842. DOI: https://doi.org/10.1111/j.1469-7610.1992. tb01959.x

[20] B. L. White, P. Castle, R. Held, Observations on the development of visually-directed reaching. Child development, (1964) 349-364. DOI: https://doi.org/10. 2307/1126701

[21] R. J. Bootsma, C. L. E. Peper,Predictive visual information sources for the regulation of action with special emphasis on catching and hitting. In Advances in psychology 85 (1992) 285-314. North-Holland. DOI: https://doi.org/10.1016/S0166-4115(08)62019-1

[22] A. Azid, H. Juahir, M. T. Latif, S. M. Zain, M. R. Osman, Feed-forward artificial neural network model for air pollutant index prediction in the southern region of Peninsular Malaysia. Journal of Environmental Protection, 4 (12A) 2013. DOI: https://doi.org/10.4236/ jep.2013.412A1001

[23] M. Van Selst, P. Jolicoeur, A solution to the effect of sample size on outlier elimination. The Quarterly Journal of Experimental Psychology Section A, 47 (3) (1994) 631-650. DOI: https://doi.org/10.1080/1464074 9408401131

[24] E. Diday, J. C. Simon, Clustering analysis. In Digital pattern recognition (1976) 47-94. Springer, Berlin, Heidelberg. DOI: https://doi.org/10.1007/9783-642-96303-2_3

[25] F. Toure, M. Badri, L. Lamontagne, A metrics suite for JUnit test code: a multiple case study on open source software. Journal of Software Engineering Research and Development, 2 (1) (2014) 1-32. DOI: https://doi.org/10.1186/s40411-014-0014-6

[26] W. D. Fisher, On grouping for maximum homogeneity. Journal of the American statistical Association, 53 (284) (1958) 789-798.

[27] S. M. Mat-Rasid, M. R. Abdullah, H. Juahir, R. M. Musa, A. B.H. Maliki, A. Adnan, N. A. Kosni, V. Eswaramoorthi, N. Alias, Relative age effect in physical attributes and motor fitness at different birthmonth quartile. Journal of Fundamental and Applied Sciences, 9 (2S) (2017) 521-538. DOI: https://doi.org/ 10.4314/jfas.v9i2s.34

[28] M. Paliwal, U. A. Kumar, Neural networks and statistical techniques: A review of applications. Expert systems with applications, 36 (1) (2009) 2-17. DOI: https://doi.org/10.1016/j.eswa.2007.10.005

[29] M. R. Abdullah, A. B. H. Musawi Maliki, R. M. Musa, N. A. Kosni, H. Juahir, M. Haque, MultiHierarchical Pattern Recognition of Athlete's Relative Performance as A Criterion for Predicting Potential Athletes. Journal of Young Pharmacists, 8 (4) (2016). DOI: 10.5530/jyp.2016.4.24

[30] B. Zupan, J. Demsar, Open-source tools for data mining. Clinics in laboratory medicine, 28 (1) (2008) 37-54. DOI: https://doi.org/10.1016/j.cll.2007.10.002

[31] N. Howard, N. Chouikhi, A. Adeel, K. Dial, A Howard, A. Hussain, BrainOS: A Novel Artificial Brain-Alike Automatic Machine Learning Framework. Frontiers in computational neuroscience, 14 (2020) 16. DOI: https://doi.org/10. 3389/fncom.2020.00016

[32] J. P. Piek, L. Dawson, L. M. Smith, N. Gasson, The role of early fine and gross motor development on later motor and cognitive ability. Human movement 
science, 27 (5) (2008) 668-681. DOI: https://doi.org/10. 1016/j.humov.2007.11.002

[33] E. Thelen, Motor development as foundation and future of developmental psychology. International journal of behavioral development, 24 (4) (2000) 385397.

[34] J. J. Campos, D. I. Anderson, M. A. Barbu-Roth, E. M. Hubbard, M. J. Hertenstein, D. Witherington, Travel broadens the mind. Infancy, 1 (2) (2000) 149219.

[35] E. Thelen, Many roads lead to Rome: Locomotion and dynamics. Infancy, 1 (2) (2000) 221-224. 